\title{
ENERGY DEPENDENT POTENTIAL PROBLEMS FOR THE ONE DIMENSIONAL $p$-LAPLACIAN OPERATOR
}

\author{
HIKMET KOYUNBAKAN, JUAN PABLO PINASCO, CRISTIAN SCAROLA
}

\begin{abstract}
In this work we analyze a nonlinear eigenvalue problem for the $p$ Laplacian operator with zero Dirichlet boundary conditions. We assume that the problem has a potential which depends on the eigenvalue parameter, and we show that, for $n$ big enough, there exists a real eigenvalue $\lambda_{n}$, and they corresponding eigenfunctions have exactly $n$ nodal domains.

We characterize the asymptotic behavior of these eigenvalues, obtaining two terms in the asymptotic expansion of $\lambda_{n}$ in powers of $n$.

Finally, we study the inverse nodal problem in the case of energy dependent potentials, showing that some subset of the zeros of the corresponding eigenfunctions is enough to determine the main term of the potential.
\end{abstract}

\section{INTRODUCTION}

In this work we consider the following problem

$$
-\left(\left|u^{\prime}\right|^{p-2} u^{\prime}\right)^{\prime}=[\lambda+g(x, \lambda)]|u|^{p-2} u, \quad x \in(0,1)
$$

with zero Dirichlet boundary conditions

$$
u(0)=u(1)=0,
$$

where $1<p<\infty, \lambda$ is a real parameter, and $g:[0,1] \times[0,+\infty) \rightarrow \mathbb{R}$ satisfies the following conditions:

H1) $g$ is a continuous function in $[0,1] \times \mathbb{R}$, and it is locally Lipschitz continuous on the variable $\lambda$ except perhaps at $\lambda=0$.

H2) There exists $C>0, \alpha \in(0,1)$ such that $|g(x, \lambda)| \leq C\left(\lambda^{\alpha}+1\right)$ for any $\lambda \in \mathbb{R}$ and $x \in[0,1]$.

We are interested in the existence of eigenvalues, and these hypotheses are enough to show the existence of an increasing sequence. However, in order to study the asymptotic behavior of the eigenvalues and the inverse nodal problem, we impose an additional condition,

H3) There exists a continuous function $h(x)$ such that $g(x, \lambda) / \lambda^{\alpha} \rightarrow h(x)$ as $\lambda \rightarrow \infty$, uniformly in $x$.

This kind of problems belong to the class of nonlinear eigenvalue problems, where the coefficients of the differential equations depend on the eigenvalue parameter. There exists several particular cases of interest, like the quadratic eigenvalue problems, see [32, 34]; or the energy dependent potentials, see $[1,10,13]$.

2000 Mathematics Subject Classification. 34A55, 34B24, 34L20, 34L30.

Key words and phrases. asymptotic behavior, eigenvalues, nodal inverse problem. 
When $p=2$, the eigenvalue problem (1.1)-(1.2) is very important in both classical and quantum mechanics. For example, such problems arise in solving KleinGordon equations which describe the motion of massless particles such as photons, they are also used for modelling vibrations of mechanical systems in viscous media, or in hydrodynamic stability problems, see [5]. In particular, generalized one dimensional Schrödinger equations with quadratic operator pencils,

$$
-u^{\prime \prime}=\left(\lambda^{2}-2 \lambda h(x)-q(x)\right) u
$$

where studied in several papers, see $[1,2,3,10,22,28,35,36]$, among other works.

The existence of a discrete set of eigenvalues in the linear case is a difficult problem, and it was solved first by Friedman and Shinbrot [11] using functional analytic techniques for linear compact symmetric operators which can be applied to the inverses of the differential operators. In the one-dimensional case, the work of Greenberg and Babuska [12] uses the shooting method as a way to obtain the eigenvalues; however, this need some monotonicity assumptions on the coefficients. Again in the linear one-dimensional case corresponding to equation (1.3), the discreteness of the spectrum and the existence of a double sequence of real eigenvalues going to $\pm \infty$ can be found in $[14,15]$, where additional conditions are imposed on the coefficients, namely,

G1) $h \in H^{1}(0,1)$.

G2) $q \in L^{2}(0,1)$ satisfies $\int_{0}^{1}\left|u^{\prime}\right|^{2}+q(x)|u|^{2} d x>0$ for $u \in H^{2}(0,1), u \neq 0$.

Without these conditions, a finite number of complex eigenvalues can appear, see also the work of Browne and Watson [2], where this question is discussed in detail.

Let us remark that this double sequence depend on the particular problem considered. In some cases, equation (1.3) appears as

$$
-u^{\prime \prime}=(\lambda-2 \sqrt{\lambda} h(x)-q(x)) u,
$$

and only positive values of $\lambda$ are allowed as eigenvalues. We prefer to work here with this type of problem, since the other case can be handled in much the same way. Let us remark that some pathologies can occur, like the existence of complex eigenvalues, or the existence of two values of $\lambda$ with the same associated eigenfunctions. We will discuss these questions briefly at the end of Section $\S 2$.

The existence of eigenvalues for the $p$-Laplacian is subtle, since the tools used for $p=2$ are not available in the quasilinear case. Also, a general nonlinearity like $g(x, \lambda)$ forbids the use of the Prüfer transform based on the generalized trigonometric functions (as in $[7,8,12]$ ) without additional conditions of monotonicity on $g$. Nevertheless, we can show that, for sufficiently big $n$, we have at least one eigenvalue $\lambda_{n}$, and the corresponding eigenfunctions have exactly $n+1$ zeros in $[0,1]$. Our first results in this direction are the following theorems:

Theorem 1.1. Let $g$ be a function satisfying $(H 1)-(H 2)$. Then there exists $n_{0} \geq 1$ such that, if $n \geq n_{0}$, there exists an eigenvalue $\lambda_{n}$ of problem (1.1)-(1.2) such that the associated eigenfunction $u_{n}$ has exactly $n+1$ zeros in $[0,1]$.

Let us observe that this theorem does not preclude the existence of more than two eigenvalues with same number of zeros. The following corollary is a direct consequence of the previous theorem and the Sturmian comparison theorem (see Theorem 2.2 in Section $\S 2$ ). 
Corollary 1.1. Let $g$ be a function satisfying $(H 1)-(H 2)$, and let us assume that $g(x, \lambda)$ is monotonic in $\lambda$. Then there exists $n_{0} \geq 1$ such that, if $n \geq n_{0}$, there exists an eigenvalue $\lambda_{n}$ of problem (1.1)-(1.2) such that the associated eigenfunction $u_{n}$ has exactly $n+1$ zeros in $[0,1]$, and there are no other eigenvalues in $\left(\lambda_{n_{0}},+\infty\right)$ such that the associated eigenfunction has $n+1$ zeros in $[0,1]$.

Also, we can compute the asymptotic expansion of $\lambda_{n}$. We obtain two terms, with a different error term depending on the relationship between $\alpha$ and $p^{\prime}$, the Sobolev conjugate exponent of $p$ satisfying

$$
\frac{1}{p}+\frac{1}{p^{\prime}}=1
$$

Theorem 1.2. Let us consider the problem (1.1)-(1.2), and suppose that $g$ satisfies conditions $H 1), H 2$ ), and $H 3)$. Then, if $\alpha p^{\prime}<1$,

$$
\lambda_{n}=n^{p} \pi_{p}^{p}-\frac{1}{p}\left(\pi_{p} n\right)^{1+\alpha p-p} \int_{0}^{1} h(x) d x+O\left(n^{2 p \alpha-2 p+1)}\right) .
$$

and, if $\alpha p^{\prime} \geq 1$,

$$
\lambda_{n}=n^{p} \pi_{p}^{p}-\frac{1}{p}\left(\pi_{p} n\right)^{1+\alpha p-p} \int_{0}^{1} h(x) d x+o\left(n^{1+\alpha p-p}\right) .
$$

Here, $\pi_{p}$ is the first nontrivial zero of the generalized $p$-sine function, see Section $\S 2$ for the corresponding definition.

Let us remark that Theorem 1.2 improves the results obtained for the quadratic eigenvalue problem and $p=2$ in [2], since an additional term is obtained. Also, less regularity is assumed on $g$ than the one imposed by hypothesis $G 1$ ) in [14]. On the other hand, assuming more regularity on the function $h$ as in $G 1$ ), more terms in the asymptotic expansion of $\lambda_{n}$ were obtained in $[14,15]$. Our proofs are based on simple arguments, depending on the classical comparison and oscillation theorems, combined with a fixed point argument.

Our next result shows that, for the quadratic eigenvalue problem, some infinite subset of the zeros of $\left\{u_{n}\right\}_{n \geq n_{0}}$ is enough to determine the function accompanying the higher order power of $\lambda$ in the potential, which enable us to determine $h$ in H3). We postpone the definition of a dense twin subset to Section $\S 5$.

Theorem 1.3. Let us consider the problem (1.1)-(1.2), and suppose that $g$ satisfies conditions H1), H2), and H3). Let $\left\{\lambda_{n}\right\}_{n \geq n_{0}}$ be the sequence of eigenvalues given by Theorem 1.1. Then, given the zeros of the corresponding eigenfunctions, we can determine univocally the function $h$. Moreover, it is enough to consider a dense twin subset of the zeros, and

$$
\begin{aligned}
h(x) & =\lim _{n \rightarrow \infty} \lambda_{n}^{-\alpha}\left[\frac{\pi_{p}^{p}}{\left(x_{j}^{n}-x_{j-1}^{n}\right)^{p}}-\lambda_{n}\right] \\
& =\lim _{n \rightarrow \infty} \pi_{p}^{p-p \alpha} n^{-p \alpha}\left[\frac{1}{\left(x_{j}^{n}-x_{j-1}^{n}\right)^{p}}-n^{p}\right] .
\end{aligned}
$$

In particular, for the quadratic eigenvalue problem, we have the following corollary, which includes the order of convergence:

Corollary 1.2. Let us consider the problem

$$
-\left(\left|u^{\prime}\right|^{p-2} u^{\prime}\right)^{\prime}=\left[\lambda+\lambda^{\alpha} h(x)+q(x)\right]|u|^{p-2} u, \quad x \in(0,1)
$$


with zero Dirichlet boundary conditions $u(0)=u(1)=0$. Then we can recover $h$ given a dense twin subset of the zeros of eigenfunctions. Moreover, if $h$ is Lipschitz continuous with constant $K$, we have

$$
\left|h(x)-\lambda_{n}^{-\alpha}\left[\frac{\pi_{p}^{p}}{\left(x_{j}^{n}-x_{j-1}^{n}\right)^{p}}-\lambda_{n}\right]\right| \leq K \delta_{n}
$$

and

$$
\left|h(x)-\pi_{p}^{p-p \alpha} n^{-p \alpha}\left[\frac{1}{\left(x_{j}^{n}-x_{j-1}^{n}\right)^{p}}-n^{p}\right]\right| \leq K \delta_{n}+O\left(n^{1-p}\right),
$$

where $\delta_{n}=\max \left\{\left|x-x_{j-1}^{n}\right|,\left|x-x_{j}^{n}\right|\right\}$.

Let us remark that the constant in the term $O\left(n^{1-p}\right)$ depends only on the $L^{\infty}$ norm of $h$ (in order to bound the integral of $h$ ), and in this case also the constant $K$ can be estimated explicitly.

The spectral inverse problems for energy dependent potentials started with the works of Jaulent and Jean [19, 20, 21], and was studied later in [3, 18, 27, 37, 36].

Let us mention some previous works in the inverse nodal problem, and let us compare briefly the differences with our proof of Theorem 1.3. First, let us recall that there are two different inverse problems for the equation

$$
-u^{\prime \prime}=\lambda r(x) u+q(x) u,
$$

one is to determine the weight $r$, and the other one is to determine the potential $q$. For quadratic eigenvalue problems,

$$
-u^{\prime \prime}=\lambda^{2} u+\lambda h(x) u+q(x) u,
$$

both $h$ and $q$ are terms of the potential, and were determined in the linear case $p=2$ by Buterin and Shieh, see [3], and by Yang in [36] in terms of a dense subset of nodes and the integrals of $h$ and $q$. Their proofs are based on precise estimates on the location of zeros of eigenfunctions obtained from the regularized traces of the pencil of operators and the corresponding Weyl function. Let us remark that only $q$ was determined before for the $p$-Laplacian operator, see the works of Goktas, Gulsen, Yilmaz, and Koyunbakan [13, 22, 23, 38].

The determination of $q$ followed the ideas introduced originally by McLaughlin in [26], which were quickly extended in $[6,16,17,24]$ among several other works. Their proofs need a detailed knowledge of the lengths of the nodal domains in terms of the eigenvalues, which depends on a better estimate on the eigenvalues than the one given by the estimate (1.4).

This method works well also in the determination of the weight $r$, and different approaches were proposed in [31], based in the problem of moments combined with integration rules on the zeros of the eigenfunctions; in [25], where the authors used the WKB method; and recently in $[29,30]$, based on the convergence of a sequence of measures concentrated on the zeros of the eigenfunctions.

Here, we are mainly interested on the characterization of $h$. The previous methods need much more information on the eigenvalues than the one available here, and the last one cannot be used in this problem since the sequence of measures converge to the Lebesgue measure, and therefore the information on $h$ and $q$ is lost. 
So, we introduce a different approach here, of more elemental character. Compared with previous works in the linear case (i.e., $[3,36]$ ), we are using more information about the nodes, since they assume only that a dense subset of zeros is given, and also it is enough to know them on more than half the interval. On the other hand, we are not assuming that the integral of $q$ and $h$ are given, and only the case $\alpha=1 / 2$ was studied in these works.

1.1. Organization of the paper. The paper is structured as follows: in Section $\S 2$ we introduce some notation and previous results. In Section $\S 3$ we prove Theorem 1.1 , and in Section $\S 4$ we prove Theorem 1.2. Section $\S 5$ is devoted to the nodal inverse problem.

\section{Preliminaries}

In this section we will recall some necessary definitions and results about solutions to the problem (1.1)-(1.2).

2.1. The one dimensional $p$-Laplacian problem. Let us start with the constant coefficient problem

$$
-\left(\left|u^{\prime}\right|^{(p-2)} u^{\prime}\right)^{\prime}=(p-1)|u|^{(p-2)} u,
$$

and let $S_{p}(x)$ be the unique solution of the initial boundary value problem satisfying $u(0)=0, u^{\prime}(0)=1$. This function is known as the generalized sine function, and for $p=2$ become $\sin x$, see $[7,8]$. The first zero of $S_{p}(x)$ is

$$
\pi_{p}=2 \int_{0}^{1} \frac{d t}{\sqrt[p]{1-t^{p}}},
$$

and enables us to characterize the eigenvalues and eigenfunctions of the problem

$$
-\left(\left|u^{\prime}\right|^{(p-2)} u^{\prime}\right)^{\prime}=(p-1) \Lambda|u|^{(p-2)} u
$$

with zero Dirichlet boundary conditions $u(0)=u(1)=0$ (see [8]). There exists a sequence of eigenvalues $\left\{\lambda_{n}\right\}_{n \geq 1}$, such that

$$
\Lambda_{1}<\Lambda_{2}<\cdots<\Lambda_{n}<\ldots \rightarrow \infty,
$$

where $\Lambda_{n}=n^{p} \pi_{p}^{p}$, and the corresponding eigenfunction is $u_{n}(x)=S_{p}\left(n \pi_{p} x\right)$. Let us note that $u_{n}$ has $n+1$ zeros in $[0,1]$, or nodal points, which define $n$ nodal domains, i. e., the interval between two consecutive zeros.

Using the shooting method and the Prufer transform, it is possible to extend this result to the nonconstant coefficient case, obtaining also estimates on the eigenvalues. An alternative procedure is the variational method, we have again a sequence of discrete eigenvalues, and the eigenfunction associated to $\Lambda_{n}$ has $n+1$ zeros or $n$ nodal domains.

We recall the following results on the existence of eigenvalues together with the fundamental theorems of comparison and oscillation for Sturm-Liouville problems, which hold for linear and quasilinear problems, and will be needed later:

Theorem 2.1. Let us assume that $g(x, \lambda)=g(x)$ does not depend on $\lambda$. Then all the eigenvalues $\Lambda$ of the problem

$$
-\left(\left|u^{\prime}\right|^{(p-2)} u^{\prime}\right)^{\prime}=(\Lambda+g(x))|u|^{(p-2)} u
$$


with zero Dirichlet boundary conditions are given by an unbounded increasing sequence $\left\{\Lambda_{n}\right\}_{n \geq 1}$, and their asymptotic behavior as $n \rightarrow \infty$ is given by

$$
\Lambda_{n}^{1 / p}=n \pi_{p}+o(n) .
$$

Any eigenfunction associated to $\Lambda_{n}$ has exactly $n+1$ nodal points in $[0,1]$, and the zeros of the eigenfunctions interlace, excluding the ones at 0 and 1.

Moreover, by considering the Sobolev space $W_{0}^{1, p}(0,1)$, we can obtain the eigenvalues by using a minimax characterization,

$$
\Lambda_{n}=\inf _{C_{n} \in \mathcal{C}_{n}} \sup _{\left\{u \in C_{n}:\|u\|_{\left.L^{p}=1\right\}}\right.} \int_{0}^{1}\left|u^{\prime}\right|^{p} d x+\int_{0}^{1} g(x)|u|^{p} d x,
$$

where $\mathcal{C}_{k}$ is the class of compact symmetric $(C=-C)$ subsets of $W_{0}^{1, p}(0,1)$ of (Krasnoselskii) genus greater or equal than $n$.

Theorem 2.2. Given two equations

$$
\begin{aligned}
& -\left(\left|u^{\prime}\right|^{(p-2)} u^{\prime}\right)^{\prime}=g_{1}(x)|u|^{(p-2)} u \\
& -\left(\left|u^{\prime}\right|^{(p-2)} u^{\prime}\right)^{\prime}=g_{2}(x)|u|^{(p-2)} u
\end{aligned}
$$

such that $g_{1}(x)<g_{2}(x)$ on $[0,1]$, then between every two nodal points of any non trivial solution of the first equation there is at least one node of every solution of the second equation.

For a proof of these results, and related ones, we refer the interested reader to $[4,7,8,9,10,33]$. Let us remark that there are similar results for Neumann and mixed boundary conditions.

2.2. Quadratic eigenvalue problems. As mentioned in the introduction, changing the spectral parameter $\sqrt{\lambda} \rightarrow \Lambda$ gives a potential of the form

$$
\Lambda^{2}-2 h(x) \Lambda-q(x),
$$

and we can expect the existence of two sequences of eigenvalues going to $\pm \infty$. Of course, in the original variable we only consider the positive values of $\Lambda$, or we need to introduce complex eigenvalues; we prefer this because the situation is harder for arbitrary powers $\lambda^{\alpha}$.

The following simple example shows the problems involved and the necessity of hypothesis $G 2)$ in $[14,15]$.

Example 2.1. Let us consider the equation $(1.3)$ in $(0,1)$, with constant coefficients $h$ and $q$,

$$
-u^{\prime \prime}=(\lambda-2 b \sqrt{\lambda}-a) u
$$

where $a$ and $b$ are real numbers.

By considering $\lambda^{2}+2 b \lambda+a=n^{2} \pi^{2}$, we get that this equation has two real solutions if and only if $a \leq b^{2}+n^{2} \pi^{2}$. However, we can have two different positive values for $\lambda_{n}$, both with the same associated eigenfunction. For example, for $b=2 \pi$, and $a=-4 \pi^{2}$, we have

$$
-u^{\prime \prime}=\left(\lambda-4 \pi \sqrt{\lambda}+4 \pi^{2}\right) u,
$$

and $u_{\lambda}(x)=\sin (\pi x)$ is the associated eigenfunction to $\sqrt{\lambda}=\pi$ and also to $\sqrt{\lambda}=3 \pi$. 


\section{Existence OF EIGENVALUES}

In this section we prove Theorem 1.1 and Corollary 1.1. The proof of Theorem 1.1 is structured in three parts: first we derive an estimate for $n_{0}$ and we define, for $n \geq n_{0}$, some intervals $I_{n}$; then, we introduce an operator $T: I_{n} \rightarrow \mathbb{R}$ which gives the $n$-th. eigenvalue of an auxiliary problem with $g(x, \lambda)$ replaced by $g(x, \mu)$, and we prove its continuity; finally, we show that there exists a fixed point $T\left(\mu_{n}\right)=\mu_{n}$, which is the eigenvalue $\lambda_{n}$.

Of course, we cannot prove that there exists a unique fixed point $\mu_{n}$ for each $n$. However, it is easy to see that this can be done by imposing a monotonicity condition on $g$ as a function of $\lambda$ as in Corollary 1.1, and its proof follows directly from the Sturmian Comparison Theorem 2.2.

\section{Proof of Theorem 1.1 .}

(1) A priori bounds on $\lambda_{n}$ and $n_{0}$.

Let us recall hypothesis $\mathrm{H} 2$ ),

$$
\lambda-C\left(\lambda^{\alpha}+1\right) \leq \lambda+g \leq \lambda+C\left(\lambda^{\alpha}+1\right),
$$

and hence, since $\alpha<1$, there exists a minimum positive integer $n_{0}$ such that, for $n \geq n_{0}$, we have

$$
\frac{C\left(\left[2 \pi_{p}^{p}(n+1)^{p}\right]^{\alpha}+1\right)}{\pi_{p}^{p}(n-1)^{p} / 2}<\frac{1}{2} .
$$

Now, for any $n \geq n_{0}$ we define $I_{n}=\left[\pi_{p}^{p}(n-1)^{p} / 2,2 \pi_{p}^{p}(n+1)^{p}\right]$. For $\lambda \in I_{n}$ we have

$$
\begin{aligned}
\frac{|g(x, \lambda)|}{\lambda} & \leq \frac{C\left(\lambda^{\alpha}+1\right)}{\lambda} \\
& \leq \frac{C\left(\left[\pi_{p}^{p}(n+1)^{p}\right]^{\alpha}+1\right)}{\pi_{p}^{p}(n-1)^{p}} \\
& <\frac{1}{2} .
\end{aligned}
$$

In other words, whenever $\lambda \in I_{n}$,

$$
|g(x, \lambda)|<\frac{\lambda}{2}
$$

(2) An auxiliary eigenvalue problem.

Now, for each $n \geq n_{0}$, and the intervals $I_{n}$ introduced above, let us define $T_{n}: I_{n} \rightarrow \mathbb{R}$, where $T_{n}(\mu)$ is the $n$-th eigenvalue of the problem

$$
-\left(\left|v^{\prime}\right|^{p-2} v^{\prime}\right)^{\prime}=[\lambda+g(x, \mu)]|v|^{p-2} v, \quad x \in(0,1)
$$

with zero Dirichlet boundary conditions. Let us recall that the associated eigenfunction has exactly $n+1$ zeros on $I_{n}$. We show now that $T_{n}$ is a continuous function.

Let $\varepsilon$ be an arbitrary positive number. Given any pair of values $\mu_{1}, \mu_{2} \in I_{n}$, since $g$ is locally Lipschitz, there exists a fixed constant $K_{n}$ such that

$$
\left|g\left(x, \mu_{1}\right)-g\left(x, \mu_{2}\right)\right| \leq K_{n}\left|\mu_{1}-\mu_{2}\right| .
$$

Thus, there exists $\delta>0$ such that, if $\left|\mu_{1}-\mu_{2}\right| \leq \delta$, we get $\left|g\left(x, \mu_{1}\right)-g\left(x, \mu_{2}\right)\right|<\varepsilon$. 
We will use now the variational characterization of the eigenvalues given by equation (2.1). There exists a compact symmetric set $C_{n}^{(1)}$ such that

$$
\lambda_{n}\left(\mu_{1}\right) \leq \sup _{\left\{u \in C_{n}^{(1)}:\|u\|_{\left.L^{p}=1\right\}}\right.} \int_{0}^{1}\left|u^{\prime}\right|^{p} d x+\int_{0}^{1} g(x)|u|^{p} d x \leq \lambda_{n}\left(\mu_{1}\right)+\varepsilon .
$$

Therefore, for any function $u \in C_{n}^{(1)}$, and $\mu_{2}$ satisfying $\left|\mu_{1}-\mu_{2}\right| \leq \delta$, we have

$$
\begin{aligned}
& \lambda_{n}\left(\mu_{2}\right)= \inf _{C_{n}} \sup _{\left\{u \in C_{n}:\|u\|_{\left.L^{p}=1\right\}}\right.}\left(\int_{0}^{1}\left|u^{\prime}\right|{ }^{p} d x+\int_{0}^{1} g\left(x, \mu_{2}\right)|u|^{p} d x\right) \\
& \leq \sup _{\left\{u \in C_{n}^{(1)}:\|u\|_{\left.L^{p}=1\right\}}\right.}\left(\int_{0}^{1}\left|u^{\prime}\right|^{p} d x+\int_{0}^{1} g\left(x, \mu_{1}\right)\right]|u|^{p} d x \\
&\left.\quad+\int_{0}^{1}\left[g\left(x, \mu_{2}\right)-g\left(x, \mu_{1}\right)\right]|u|^{p} d x\right) \\
& \leq\left(\lambda_{n}\left(\mu_{1}\right)+\varepsilon+\sup _{\left\{u \in C_{n}^{(1)}:\|u\|_{\left.L^{p}=1\right\}}\right.}\left(g\left(x, \mu_{2}\right)-g\left(x, \mu_{1}\right) \|\left. u\right|^{p} d x\right)\right. \\
& \leq \lambda_{n}\left(\mu_{1}\right)+\varepsilon+\varepsilon\|u\|_{L^{p}}^{p} \\
&=\lambda_{n}\left(\mu_{1}\right)+2 \varepsilon .
\end{aligned}
$$

Interchanging the role of $\mu_{1}$ and $\mu_{2}$, taking an auxiliary set $C_{n}^{(2)}$, we get $\lambda_{n}\left(\mu_{1}\right) \leq$ $\lambda_{n}\left(\mu_{2}\right)+2 \varepsilon$, and thus

$$
\left|\lambda_{n}\left(\mu_{1}\right)-\lambda_{n}\left(\mu_{2}\right)\right| \leq 2 \varepsilon .
$$

Hence, the operator $T_{n}: I_{n} \rightarrow \mathbb{R}$ is continuous.

(3) The fixed point argument.

Let us show that $T_{n}\left(\mu_{n}\right)=\mu_{n}$ for some $\mu_{n} \in I_{n}$. To this end, since $T_{n}$ is a continuous function, it is enough to show that:

$$
\begin{aligned}
& \text { i.- } T_{n}\left(\pi_{p}^{p}(n-1)^{p} / 2\right) \geq \pi_{p}^{p}(n-1)^{p} / 2, \\
& \text { ii.- } T_{n}\left(2 \pi_{p}^{p}(n+1)^{p}\right) \leq 2 \pi_{p}^{p}(n+1)^{p} .
\end{aligned}
$$

Let us observe that both inequalities implies $T_{n}: I_{n} \rightarrow I_{n}$, and then the classical Bolzano's theorem or the Brower Fixed Point Theorem implies the existence of a fixed point.

Let us prove $i .-$. Let us suppose that

$$
\lambda_{n}\left(\pi_{p}^{p}(n-1)^{p} / 2\right)=T_{n}\left(\pi_{p}^{p}(n-1)^{p} / 2\right)<\pi_{p}^{p}(n-1)^{p} / 2 .
$$

Then, from inequality (3.1),

$$
\begin{aligned}
\lambda_{n}\left(\pi_{p}^{p}(n-1)^{p} / 2\right)+g\left(x, \pi_{p}^{p}(n-1)^{p} / 2\right) & =T_{n}\left(\pi_{p}^{p}(n-1)^{p} / 2\right)+g\left(x, \pi_{p}^{p}(n-1)^{p} / 2\right) \\
& \leq \pi_{p}^{p}(n-1)^{p} / 2+\pi_{p}^{p}(n-1)^{p} / 4 \\
& <\pi_{p}^{p}(n-1)^{p},
\end{aligned}
$$

contradicting the Sturmian Comparison Theorem 2.2, since the corresponding eigenfunction has $n+1$ zeros, and the solution to

$$
-\left(\left|w^{\prime}\right|^{p-2} w^{\prime}\right)^{\prime}=\pi_{p}^{p}(n-1)^{p}|w|^{p-2} w, \quad x \in(0,1)
$$


satisfying $w(0)=w(1)=0$ has exactly $n$ zeros.

In much the same way we prove $i i .-$, let us suppose that

$$
\lambda_{n}\left(2 \pi_{p}^{p}(n+1)^{p}\right)=T_{n}\left(2 \pi_{p}^{p}(n+1)^{p}\right)>2 \pi_{p}^{p}(n+1)^{p},
$$

and using again inequality (3.1),

$$
\begin{aligned}
\lambda_{n}\left(2 \pi_{p}^{p}(n+1)^{p}\right)+g\left(x, 2 \pi_{p}^{p}(n+1)^{p}\right) & =T_{n}\left(2 \pi_{p}^{p}(n+1)^{p}\right)+g\left(x, 2 \pi_{p}^{p}(n+1)^{p}\right) \\
& >2 \pi_{p}^{p}(n+1)^{p}-2 \pi_{p}^{p}(n+1)^{p} / 2 \\
& =\pi_{p}^{p}(n+1)^{p},
\end{aligned}
$$

contradicting the Sturmian Comparison Theorem 2.2, since the solution to

$$
-\left(\left|w^{\prime}\right|^{p-2} w^{\prime}\right)^{\prime}=\pi_{p}^{p}(n+1)^{p}|w|^{p-2} w, \quad x \in(0,1)
$$

satisfying $w(0)=w(1)=0$ has exactly $n+2$ zeros.

We have obtained a sequence of eigenvalues $\left\{\lambda_{n}\right\}_{n \geq n_{0}}$, and their associated eigenfunctions $\left\{u_{n}\right\}_{n \geq n_{0}}$. It is clear that the eigenfunction $u_{n}$ has $n+1$ zeros in $[0,1]$, since it is the associated eigenfunction to the $n$-th eigenvalue of the auxiliary Sturm Liouville problem with Dirichlet boundary conditions. Hence, they are all different.

The proof is finished.

Remark 3.1. Let us observe that the argument cannot be strengthened to show that there are no other eigenvalues. However, this can be proved by imposing some monotonicity condition in order to ensure that $T_{n}(\mu)$ is an increasing function, as stated in Corollary 1.1.

Proof of Corollary 1.1. Let $n_{0}$ as in the proof of Theorem 1.1. For each $n \geq n_{0}$ we have an eigenvalue $\lambda_{n} \in I_{n}$, and the corresponding eigenfunction has exactly $n+1$ zeros in $[0,1]$.

Let us suppose that $\Lambda \in\left(\lambda_{n_{0}},+\infty\right), \Lambda \neq \lambda_{n}$ is another eigenvalue, and let $u_{\Lambda}$ be its associated eigenfunction. There exists some $n$ such that $\lambda_{n}<\Lambda<\lambda_{n+1}$, and the monotonicity condition on $g$ implies that

$$
\lambda_{n}+g\left(x, \lambda_{n}\right)<\Lambda+g(x, \Lambda)<\lambda_{n+1}+g\left(x, \lambda_{n+1}\right) .
$$

Therefore, the Sturmian Comparison Theorem 2.2 implies $u_{\Lambda}$ has more than $n+1$ zeros, and simultaneously, less than $n+2$, which is impossible.

Hence, $\Lambda$ must coincide with one of the eigenvalues $\lambda_{n}$. However, there exists a unique solution to the initial boundary value problem given by equation (1.1) satisfying $u(0)=0, u^{\prime}(0)=1$, so $u_{\Lambda}$ is an appropriate multiple of $u_{n}$.

The proof is finished.

\section{Asymptotic Behavior of Eigenvalues.}

Let us observe that the existence of an eigenvalue $\lambda_{n} \in I_{n}$ proved in the previous section implies that $\lambda_{n}=O\left(n^{p}\right)$. In order to prove Theorem 1.2, we need the following previous results.

Lemma 4.1. Let $\left\{\lambda_{n}\right\}_{n \geq n_{0}}$ be the sequence of eigenvalues of problem (1.1)-(1.2) obtained in Theorem 1.1, where $g$ satisfies H1) and H2). Then

$$
\lambda_{n}=n^{p} \pi_{p}^{p}+o\left(n^{p}\right) .
$$


Proof. Let us take $\varepsilon>0$. Let us write equation (1.1) as

$$
-\left(\left|u^{\prime}\right|^{p-2} u^{\prime}\right)^{\prime}=\lambda\left[1+\frac{g(x, \lambda)}{\lambda}\right]|u|^{p-2} u .
$$

Using condition $H 1$ ) we get

$$
1-C\left[\lambda^{\alpha-1}-\lambda^{-1}\right] \leq 1+\frac{g(x, \lambda)}{\lambda} \leq 1+C\left[\lambda^{\alpha-1}-\lambda^{-1}\right]
$$

and hence $1-\varepsilon \leq 1+\frac{g(x, \lambda)}{\lambda} \leq 1+\varepsilon$ if $\lambda$ is big enough.

By using the Sturmian Comparison Theorem 2.2,

$$
(1-\varepsilon) \pi_{p}^{p} n^{p} \leq \lambda_{n} \leq(1+\varepsilon) \pi_{p}^{p} n^{p},
$$

and the Lemma is proved.

Lemma 4.2. Let $\left\{u_{n}\right\}_{n>n_{0}}$ be the eigenfunctions associated to $\left\{\lambda_{n}\right\}_{n} \geq n_{0}$, the eigenvalues of problem (1.1)-(1.2) obtained in Theorem 1.1, where $g$ satisfies $H 1)$ and H2). Let $0=x_{0}<x_{1}<\cdots<x_{n}=1$ be the zeros of $u_{n}$. Then, for $1 \leq j \leq n$, there exists some $y_{j} \in\left[x_{j-1}, x_{j}\right]$ such that

$$
\lambda_{n}+g\left(y_{j}, \lambda_{n}\right)=\frac{\pi_{p}^{p}}{\left(x_{j}-x_{j-1}\right)^{p}} .
$$

Proof. By re-scaling the interval, we know that $\pi_{p}^{p} /\left(x_{j}-x_{j-1}\right)^{p}$ is the first eigenvalue of the $p$-Laplacian operator in $\left[x_{j-1}, x_{j}\right]$. Now, the Sturmian Comparison Theorem 2.2 implies

$$
\begin{gathered}
\frac{\pi_{p}^{p}}{\left(x_{j}-x_{j-1}\right)^{p}} \geq \min _{y \in\left[x_{j-1}, x_{j}\right]} \lambda_{n}+g\left(y, \lambda_{n}\right), \\
\frac{\pi_{p}^{p}}{\left(x_{j}-x_{j-1}\right)^{p}} \leq \max _{y \in\left[x_{j-1}, x_{j}\right]} \lambda_{n}+g\left(y, \lambda_{n}\right),
\end{gathered}
$$

and the result follows by using the continuity of $g$ and Bolzano's Theorem.

We are ready to prove Theorem 1.2

Proof of Theorem 1.2. Applying Lemma 4.2 in the nodal domains of $u_{n}$, and the Taylor's expansion $(1+z)^{1 / p}=1+z / p+O\left(z^{2}\right)$ for $z \approx 0$, we get

$$
\begin{aligned}
\frac{\pi_{p}}{x_{j}-x_{j-1}} & =\lambda_{n}^{1 / p}\left(1+\frac{g\left(y_{j}, \lambda_{n}\right)}{\lambda_{n}}\right)^{1 / p} \\
& =\lambda_{n}^{1 / p}\left(1+\frac{g\left(y_{j}, \lambda_{n}\right)}{p \lambda_{n}}+O\left(\lambda_{n}^{2 \alpha-2}\right)\right) \\
& =\lambda_{n}^{1 / p}+\frac{g\left(y_{j}, \lambda_{n}\right)}{p \lambda_{n}^{(p-1) / p}}+O\left(\lambda_{n}^{2 \alpha-2+1 / p}\right) .
\end{aligned}
$$


Now, by multiplying both sides by $\left(x_{j}-x_{j-1}\right)$ and summing from 1 to $n$, we get

$$
\begin{aligned}
n \pi_{p} & =\sum_{j=1}^{n} \pi_{p} \\
& =\sum_{j=1}^{n}\left(\lambda_{n}^{1 / p}\left(x_{j}-x_{j-1}\right)+\frac{g\left(y_{j}, \lambda_{n}\right)}{p \lambda_{n}^{(p-1) / p}}\left(x_{j}-x_{j-1}\right)\right)+O\left(\lambda_{n}^{2 \alpha-2+1 / p}\right) \\
& =\lambda_{n}^{1 / p}+\frac{1}{p \lambda_{n}^{(p-1) / p}} \sum_{j=1}^{n} g\left(y_{j}, \lambda_{n}\right)\left(x_{j}-x_{j-1}\right)+O\left(\lambda_{n}^{2 \alpha-2+1 / p}\right),
\end{aligned}
$$

Let us observe that the term $O\left(\lambda_{n}^{2 \alpha-2+1 / p}\right)$ in each interval can be bounded independently of $j$ by the same constant depending only on $C$ given by condition H1), so after multiplying by $\left(x_{j}-x_{j-1}\right)$ and summing up, we recover the same term since the total lengths of the intervals is equal to one.

Now, using that

$$
\lim _{n \rightarrow \infty} \lambda_{n}^{-\alpha} \sum_{j=1}^{n} g\left(y_{j}, \lambda_{n}\right)\left(x_{j}-x_{j-1}\right)=\lim _{n \rightarrow \infty} \sum_{j=1}^{n} h\left(y_{j}\right)\left(x_{j}-x_{j-1}\right)=\int_{0}^{1} h(x) d x,
$$

we get

$$
n \pi_{p}=\lambda_{n}+\frac{1}{p \lambda_{n}^{(p-1) / p-\alpha}} \int_{0}^{1} h(x) d x+o\left(\lambda_{n}^{\alpha-(p-1) / p}\right)+O\left(\lambda_{n}^{2 \alpha-2+1 / p}\right),
$$

where the $o($.$) term comes from the error between the integral and the corresponding$ Riemann' sum, and the error between $g(x, \lambda) \lambda^{-\alpha}$ and $h(x)$.

Let us compare the exponent on the error terms. Since $\alpha<1$, we have that

$$
\alpha-(p-1) / p>2 \alpha-2+1 / p .
$$

This implies that the error term is $o\left(\lambda_{n}^{\alpha-(p-1) / p}\right)$ when $\alpha p^{\prime} \geq 1$, since the exponent is positive, and it is $O\left(\lambda_{n}^{2 \alpha-2+1 / p}\right)$ in the other case.

Finally, by replacing $\lambda_{n}=\pi_{p}^{p} n^{p}+o\left(n^{p}\right)$ from Lemma 4.1, the proof is finished.

\section{NodAl InVERSE PROBlem}

In order to study the nodal inverse problem we introduce the following definitions:

Definition 5.1. Let us call $X_{g}$ the set of zeros of the eigenfunctions of problem (1.1)-(1.2), that is,

$$
X_{g}=\left\{x_{j}^{n}: 0 \leq j \leq n, n \geq n_{0}\right\} .
$$

Definition 5.2. A dense twin subset of zeros $T X_{g}$ is a subset of $X_{g}$ satisfying that, for any $x \in[0,1]$, there exists a sequence of pair of consecutive zeros in $T X_{g}$, that is, $\left\{\left(x_{j-1}^{n}, x_{j}^{n}\right)\right\}_{n \geq n_{0}} \subset T X_{g}$, such that $x_{j-1}^{n}, x_{j}^{n} \rightarrow x$ as $n \rightarrow \infty$.

Remark 5.1. Let us note that the existence of this kind of sets is given by Lemma 4.2 , since $\lambda_{n}=O\left(n^{p}\right)$ implies that the distance between consecutive zeros must vanish. 
Proof of Theorem 1.3. Let us fix $x \in[0,1]$, and some $\varepsilon>0$ sufficiently small. Let us consider the corresponding sequence of zeros $\left\{\left(x_{j-1}^{n}, x_{j}^{n}\right)\right\}_{n \geq n_{0}}$ in the dense twin subset.

Proceeding as in the proof of Theorem 1.2, by applying Lemma 4.2 in the nodal domains of $u_{n}$, we get

$$
\lambda_{n}^{p}+g\left(y_{j}^{n}, \lambda_{n}\right)=\frac{\pi_{p}^{p}}{\left(x_{j}^{n}-x_{j-1}^{n}\right)^{p}} .
$$

for some $y_{j}^{n} \in\left(x_{j-1}^{n}, x_{j}^{n}\right)$. Let us observe that $y_{j}^{n} \rightarrow x$ when $n \rightarrow \infty$ as well.

Using $H 3$ ), there exists $n_{1}$ such that, if $n \geq n_{1}$, we have

$$
\left|g(\lambda, x)-\lambda^{\alpha} h(x)\right|<\varepsilon \lambda^{\alpha}
$$

for any $x \in[0,1]$. Hence,

$$
\left|\lambda_{n}^{p}+\lambda_{n}^{\alpha} h\left(y_{j}^{n}\right)-\frac{\pi_{p}^{p}}{\left(x_{j}^{n}-x_{j-1}^{n}\right)^{p}}\right|<\varepsilon \lambda_{n}^{\alpha},
$$

which implies that

$$
\left|h\left(y_{j}^{n}\right)-\lambda_{n}^{-\alpha}\left[\frac{\pi_{p}^{p}}{\left(x_{j}^{n}-x_{j-1}^{n}\right)^{p}}-\lambda_{n}\right]\right|<\varepsilon .
$$

Since $h$ is continuous (and hence, uniformly continuous in $[0,1]$ ) there exists $n_{2}$ such that

$$
\left|h(x)-h\left(y_{j}^{n}\right)\right|<\varepsilon
$$

for any $n \geq n_{2}$. Finally, adding the terms $\pm h(x)$ and using the triangular inequality, we obtain

$$
\left|h(x)-\lambda_{n}^{-\alpha}\left[\frac{\pi_{p}^{p}}{\left(x_{j}^{n}-x_{j-1}^{n}\right)^{p}}-\lambda_{n}\right]\right|<2 \varepsilon
$$

for any $n \geq \max \left\{n_{1}, n_{2}\right\}$.

This proves the first characterization of $h$ involving both the zeros and the eigenvalues. Next, we show that the eigenvalues can be replaced by their asymptotic expansion.

Using Theorem 1.2, we know that $\lambda_{n}=\left(\pi_{p} n\right)^{p}+O\left(n^{s}\right)$, with $s=1+\alpha p-$ $p<p$. Therefore, $n^{s-p} \rightarrow 0$ with $n$, and we can perform a Taylor expansion for $\left(1+O\left(n^{s-p}\right)\right)^{\alpha}$ obtaining

$$
\begin{aligned}
\lambda_{n}^{-\alpha}\left[\frac{\pi_{p}^{p}}{\left(x_{j}^{n}-x_{j-1}^{n}\right)^{p}}-\lambda_{n}\right] & =\frac{\left[\frac{\pi_{p}^{p}}{\left(x_{j}^{n}-x_{j-1}^{n}\right)^{p}}-\left(\pi_{p} n\right)^{p}\right]+O\left(n^{s}\right)}{\left[\left(\pi_{p} n\right)^{p}+O\left(n^{s}\right)\right]^{\alpha}} \\
& =\frac{\left[\frac{\pi_{p}^{p}}{\left(x_{j}^{n}-x_{j-1}^{n}\right)^{p}}-\left(\pi_{p} n\right)^{p}\right]+O\left(n^{s}\right)}{\left(\pi_{p} n\right)^{p \alpha}\left[1+O\left(n^{s-p}\right)\right]^{\alpha}} \\
& =\frac{\left[\frac{\pi_{p}^{p}}{\left(x_{j}^{n}-x_{j-1}^{n}\right)^{p}}-\left(\pi_{p} n\right)^{p}\right]+O\left(n^{s}\right)}{\left(\pi_{p} n\right)^{p \alpha}+O\left(n^{s-p+p \alpha}\right)}
\end{aligned}
$$


We can write the term in the right hand side as

$$
\left(\frac{\left[\frac{\pi_{p}^{p}}{\left(x_{j}^{n}-x_{j-1}^{n}\right)^{p}}-\left(\pi_{p} n\right)^{p}\right]+O\left(n^{s}\right)}{\left(\pi_{p} n\right)^{p \alpha}}\right)\left(\frac{\left(\pi_{p} n\right)^{p \alpha}}{\left(\pi_{p} n\right)^{p \alpha}+O\left(n^{s-p+p \alpha}\right)}\right),
$$

which is

$$
\left(\frac{\left[\frac{\pi_{p}^{p}}{\left(x_{j}^{n}-x_{j-1}^{n}\right)^{p}}-\left(\pi_{p} n\right)^{p}\right]}{\left(\pi_{p} n\right)^{p \alpha}}+O\left(n^{s-p \alpha}\right)\right)\left(1-\frac{O\left(n^{s-p+p \alpha}\right)}{\left(\pi_{p} n\right)^{p \alpha}+O\left(n^{s-p+p \alpha}\right)}\right),
$$

or, equivalently,

$$
\left(\frac{\left[\frac{\pi_{p}^{p}}{\left(x_{j}^{n}-x_{j-1}^{n}\right)^{p}}-\left(\pi_{p} n\right)^{p}\right]}{\left(\pi_{p} n\right)^{p \alpha}}+O\left(n^{s-p \alpha}\right)\right)\left(1-O\left(n^{s-p}\right)\right) .
$$

By observing that the expression converges to $h(x)$, and $n^{s-p}<n^{s-p \alpha}$, we get

$$
h(x)=\lim _{n \rightarrow \infty}\left(\left(\pi_{p} n\right)^{-p \alpha}\left[\frac{\pi_{p}^{p}}{\left(x_{j}^{n}-x_{j-1}^{n}\right)^{p}}-\left(\pi_{p} n\right)^{p}\right]+O\left(n^{s-p \alpha}\right)\right),
$$

and the theorem is proved.

The proof of Corollary 1.2 is a direct consequence of the previous computation, and only the order of convergence needs to be proved.

Proof of Corollary 1.2. Starting as before from

$$
\lambda_{n}+\lambda_{n}^{\alpha} h\left(y_{j}^{n}\right)=\frac{\pi_{p}^{p}}{\left(x_{j}^{n}-x_{j-1}^{n}\right)^{p}},
$$

where $y_{j}^{n} \in\left(x_{j-1}^{n}, x_{j}^{n}\right)$, we have

$$
\begin{aligned}
h(x) & =h(x)-h\left(y_{j}^{n}\right)+\lambda_{n}^{-\alpha}\left[\frac{\pi_{p}^{p}}{\left(x_{j}^{n}-x_{j-1}^{n}\right)^{p}}-\lambda_{n}\right] \\
& \leq \lambda_{n}^{-\alpha}\left[\frac{\pi_{p}^{p}}{\left(x_{j}^{n}-x_{j-1}^{n}\right)^{p}}-\lambda_{n}\right]+K\left|x-y_{j}^{n}\right| \\
& \leq \lambda_{n}^{-\alpha}\left[\frac{\pi_{p}^{p}}{\left(x_{j}^{n}-x_{j-1}^{n}\right)^{p}}-\lambda_{n}\right]+K \delta_{n} .
\end{aligned}
$$

From the previous proof we know that

$$
\lambda_{n}^{-\alpha}\left[\frac{\pi_{p}^{p}}{\left(x_{j}^{n}-x_{j-1}^{n}\right)^{p}}-\lambda_{n}\right]=\left[\frac{\frac{\pi_{p}^{p}}{\left(x_{j}^{n}-x_{j-1}^{n}\right)^{p}}-\left(\pi_{p} n\right)^{p}}{\left(\pi_{p} n\right)^{-p \alpha}}\right]+O\left(n^{s-p \alpha}\right) .
$$

Since $s-p \alpha=(1+p \alpha-p)-p \alpha=1-p$, we get an error term $O\left(n^{1-p}\right)$, and the proof is finished. 
Acknowledgements The first author is member of CONICET (Argentina). The second author is member of Firat University [Turkey]. The third author is a Fellow of CONICET (Argentina). This paper was partially supported by grants UBACyT 20020130100283BA, CONICET PIP $11220150100032 \mathrm{CO}$ and ANPCyT PICT 20120153.

\section{REFERENCES}

[1] E. Bairamov, O. Cakar, O. Celebi, Quadratic pencil of Schrodinger operators with spectral singularities: discrete spectrum and principal functions., J. Math. Anal. Appl. 216 (1997) 303-320.

[2] P. J. Browne, B. A. Watson, Oscillation theory for a quadratic eigenvalue problem. Quaest. Math. 31 (2008) 345-357.

[3] S. A. Buterin, C. T. Shieh, Incomplete inverse spectral and nodal problems for differential pencils. Results in Mathematics 62 (2012) 167-179.

[4] K. Chadan, D. Colton, L. Paivarinta, W. Rundell, An introduction to inverse scattering and inverse spectral problems With a foreword by Margaret Cheney. SIAM Monographs on Mathematical Modeling and Computation. Society for Industrial and Applied Mathematics (SIAM), Philadelphia, PA, 1997. x+198 pp. ISBN: 0-89871-387-0

[5] S. Chandrasekhar, Hydrodynamic stability, Clarendon Press, Oxford, 1961.

[6] X. Chen, Y.-H. Cheng, C.-K. Law, A Tikhonov regularization for the inverse nodal problem for p-Laplacian. J. Math. Anal. Appl. 395 (2012) 230-240.

[7] M. Del Pino Manuel, P. Drábek, R. Manásevich, The Fredholm alternative at the first eigenvalue for the one-dimensional p-Laplacian, J. Differential Equations, 151 (1999) 386419.

[8] P. Drábek, R. Manásevich, On the Closed Solutions to some Nonhomogeneous Eigenvalue Problems with p-Laplacian, Diff. Int. Equations. 12 (1999) 773-788.

[9] J. Fernández Bonder, J. P. Pinasco, Asymptotic Behavior of the Eigenvalues of the One Dimensional Weighted p- Laplace Operator, Arkiv för Mat. 41 (2003) 267-280.

[10] G. Freiling, V. Yurko, Inverse Sturm-Liouville problems and their applications. Nova Science Publishers, Inc., Huntington, NY, 2001. x+356 pp. ISBN: 1-56072-909-0

[11] A. Friedman, M. Shinbrot. Nonlinear eigenvalue problems. Acta Mathematica 121 (1968) $77-125$.

[12] L. Greenberg, I. Babuška, A Continuous Analogue of Sturm Sequences in the Context of Sturm-Liouville Equations. SIAM Journal on Numerical Analysis 26(4) (1989) 920-945.

[13] T. Gulsen, E. Yilmaz, Inverse nodal problem for p-Laplacian diffusion equation with polynomially dependent spectral parameter Commun. Fac. Sci. Univ. Ank. Ser. A1 Math. Stat. 65 (2016) 23-36.

[14] G. Sh. Guseinov, On the spectral analysis of a quadratic pencil of Sturm-Liouville operators. (Russian) Dokl. Akad. Nauk SSSR 285 (1985) 1292171296. English translation: Soviet Math. Dokl. 32 (1985) 859-862. [Proofs in Guseinov, G. Sh. Inverse spectral problems for a quadratic pencil of Sturm-Liouville operators on a finite interval. Spectral theory of operators and its applications, No. 7 (Russian), 51-101, "Elm", Baku, 1986.]

[15] G. Sh. Guseinov, On construction of a quadratic Sturm-Liouville operator pencil from spectral data. Proc. Inst. Math. Mech. Natl. Acad. Sci. Azerb. 40 (2014), 203-214.

[16] O. H. Hald, J. R. McLaughlin, Solution of inverse nodal problems. Inverse Problems 5 (1989) 307-347.

[17] O. H. Hald and J. R. McLaughlin, Inverse problems: recovery of BV coefficients from nodes, Inverse Problems 14 (1998) 245-273.

[18] R. Hryniv, N. Pronska, Inverse spectral problems for energy-dependent SturmLiouville equations. Inverse Problems 28 (2012) 085008.

[19] M. Jaulent, C. Jean. The inverse s-wave scattering problem for a class of potentials depending on energy. Comm. Math. Phys. 28 (1972) 177-220.

[20] M. Jaulent, C. Jean. The inverse problem for the one-dimensional Schrodinger equation with an energy-dependent potential. I. Ann. Inst. H. Poincare Sect. A (N.S.) 25 (1976) 105-118. 
[21] M. Jaulent and C. Jean. The inverse problem for the one-dimensional Schrodinger equation with an energy-dependent potential. II. Ann. Inst. H. Poincare Sect. A (N.S.) 25 (1976) 119-137.

[22] H. Koyunbakan, Inverse problem for a quadratic pencil of Sturm-Liouville operator J. Math. Anal. Appl. 378 (2011) 549-554.

[23] H. Koyunbakan, Inverse nodal problem for p-Laplacian energy-dependent Sturm-Liouville equation Bound. Value Probl. 2013 (2013) 1-8.

[24] C.K. Law, W.-C. Lian, Wei-Cheng, W.-C. Wang, The inverse nodal problem and the Ambarzumyan problem for the p-Laplacian Proc. Roy. Soc. Edinburgh Sect. A 139 (2009) 12611273.

[25] A. Martínez-Finkelshtein, P. Martínez-González, A. Zarzo. WKB approach to zero distribution of solutions of linear second order differential equations. Journal of computational and applied mathematics 145 (2002) 167-182.

[26] J.R. McLaughlin, Inverse spectral theory using nodal points as data, a uniqueness result. J. Differential Equations 73 (1988) 354-362.

[27] C. van der Mee, V. Pivovarchik, Inverse scattering for a Schrodinger equation with energy dependent potential. Journal of Mathematical Physics 42 (2001) 158-181.

[28] C. van der Mee, V. Pivovarchik. Some properties of the eigenvalues of a Schrdinger equation with energy-dependent potential. Contemp. Math. 307 (2002) 305-310.

[29] J. P. Pinasco, C. Scarola, A nodal inverse problem for second order Sturm-Liouville operators with indefinite weights. Appl. Math. Comput. 256 (2015) 819-830.

[30] J. P. Pinasco, C. Scarola, A nodal inverse problem for a quasi-linear ordinary differential equation in the half-line J. Differential Equations 261 (2016) 1000-1016.

[31] C-L. Shen, On the nodal sets of the eigenfunctions of the string equation. SIAM journal on mathematical analysis 19 (1988) 1419-1424.

[32] R. E. L. Turner, Some variational principles for a nonlinear eigenvalue problem. J. Math. Anal. Appl. 17 (1967) 151-160.

[33] W. Walter, Sturm-Liouville theory for the radial operator. Math. Z. 227 (1998) 175-185.

[34] H. F. Weinberger, On a nonlinear eigenvalue problem. J. Math. Anal. Appl. 21 (1968) 506-509.

[35] C.-F. Yang, Trace formulae for differential pencils with spectral parameter dependent boundary conditions. Math. Methods Appl. Sci. 37 (2014) 1325-1332.

[36] C.-F. Yang, An inverse problem for a differential pencil using nodal points as data. Israel Journal of Mathematics 204 (2014) 431-446.

[37] C.-F. Yang, Y. X. Guo. Determination of a differential pencil from interior spectral data. Journal of Mathematical Analysis and Applications 375 (2011) 284-293.

[38] E. Yilmaz, S. Goktas, H. Koyunbakan, On the Lipschitz stability of inverse nodal problem for p-Laplacian Schrdinger equation with energy dependent potential. Bound. Value Probl. 2015:32 (2015) 1-8.

(J.P. Pinasco) Departamento de Matemática, FCEyn,

Universidad de Buenos Aires

e imas, UBA-CONicet, Ciudad Universitaria, Pabellón I

(1428) Buenos Aires, Argentina.

E-mail address: jpinasco@dm.uba.ar

URL: http://mate.dm.uba.ar/ jpinasco

(H. K. Koyunbakan) Firat University, Faculty of Science,

Department of Mathematics,

Elazig, 23119, TURKEY.

E-mail address: hkoyunbakan@gmail.com

(C. Scarola) Departamento de Matemtica,

Facultad de Ciencias Exactas y Naturales,

Universidad NaCional de La PAMPa

Uruguay 151 (6300), Santa Rosa, La Pampa.

E-mail address: cristian.scarola@gmail.com 